\title{
EDITORIAL Prologue: Special Spotlight Issue on Japan
}

(c) The Author(s), under exclusive licence to Springer Nature Limited 2021

Journal of Human Hypertension; https://doi.org/10.1038/s41371021-00589-2

High blood pressure (BP; hypertension) is a leading global health risk. In an international survey, the prevalence of persons who had systolic BP (SBP) $\geq 140 \mathrm{~mm} \mathrm{Hg}$ increased substantially from 1990 to 2015, and deaths and losses of disability-adjusted life-years (DALYs) associated with high BP also increased [1]. The drivers of these trends include urbanization in developing countries, increased dietary salt intake, low consumption of fruits and vegetables, overweight and obesity, low physical activity, and ecosystem disruption (e.g., pollution and natural disasters). The management of hypertension at the population level must continue to strengthen as a team effort.

Evidence-based guidelines are used in health care systems to aid in treatment decisions and ensure quality and consistency in patient care throughout the world. Recommendations within guidelines are used for decision-making not only in clinical practice but also to address larger issues within health systems, including health insurance reimbursement, health policy formation, and topics across the health care continuum (e.g. health promotion, screening, diagnosis). Guidelines are established generally based on systematic reviews of healthcare interventions, diagnostic tests, prognostic markers, and cost-effective analyses. During the process, methodological rigor and involvement of multidisciplinary healthcare professionals, tailoring the recommendations to the challenges and needs of their own countries, are essential to help ensure the acceptance and successful implementation of guidelines.

BP guidelines for care are published by several internationally recognized organizations, including those from the United States, United Kingdom, Canada, China, and Japan. The quality of guidelines across countries can be assessed and compared using the Appraisal of Guidelines for Research and Evaluation (AGREE) instrument. The instrument includes 23 key items divided into 6 domains, including Scope and Purpose, Stakeholder Involvement, Rigor of Development, Clarity and Presentation, Applicability, and Editorial Independence. Elucidating the differences in BP guidelines across countries, along with identifying knowledge gaps in each, will highlight the need for reevaluation of the literature to provide relevant updates to guidelines and improve best clinical practices. By striving to achieve consensus, the global community will unite in its approach for decreasing the CVD burden of vulnerable populations.

In this special spotlight issue on Japan, each review explores similarities and differences among the latest BP management guidelines from Japan, the US, and Europe. Hisamatsu and Miura discuss differences in the prevalence, awareness, treatment, and control of hypertension between Japan vs. the US and Europe, and the reasons for the differences. Asayama et al., identify how and why the use of BP measurements in the diagnosis and treatment of hypertension differs between Japan vs. the US and Europe. Nishiyama and Tsuchihashi et al., provide an overview of the current understanding of the relationship between sodium intake and BP, including prevalence, pathophysiology, and treatment. Kario et al., discusses how and why choices regarding initial medication and BP target levels differ between Japan vs. the US and Europe. Sakima et al., discuss how to put the guideline into practice in Japan. Each of the selected reviews identifies aspects of BP management that should be discussed further in future BP guidelines worldwide, as well as the types of research that are required to advance knowledge in specific areas. Collectively, the information presented in this special issue affirms that BP guidelines are a means to building a better health system to provide better care and treatment for people-a means to an end, not an end in themselves.

Yuichiro Yano $\mathbb{D}^{1,2 \times}$, Akira Nishiyama ${ }^{3}{ }^{3}$, Toshihiko Ishimitsu ${ }^{4}$, Naoki Kashihara ${ }^{5}$, Hiromi Rakugi iD ${ }^{6}$, Kouichi Tamura ${ }^{7,8}$ Hiroshi Itoh ${ }^{9}$, Takuya Tsuchihashi ${ }^{10}$, Atsushi Sakima (D ${ }^{11}$ Hisatomi Arima (iD ${ }^{12}$, Tetsutaro Matayoshi D $^{13}$, Yutaka Imai ${ }^{14}$, Katsuyuki Miura ${ }^{15}$, Takashi Hisamatsu (D) ${ }^{16}$, Kei Asayama (D) 17,18,19' Takayoshi Ohkubo ${ }^{17}$, Kouihci Node ${ }^{20}$ and Kazuomi Kario (D) ${ }^{21}$

${ }^{1}$ Center for Novel and Exploratory Clinical Trials, Yokohama City University, Yokohama, Japan. ${ }^{2}$ Department of Family Medicine and Community Health, Duke University, Durham, NC, USA. ${ }^{3}$ Department of Pharmacology, Faculty of Medicine, Kagawa University, Kagawa, Japan. ${ }^{4}$ Department of Nephrology and Hypertension, Dokkyo Medical University, Tochigi, Japan. ${ }^{5}$ Department of Nephrology and Hypertension, Kawasaki Medical School, Okayama, Japan.

${ }^{6}$ Department of Geriatric and General Medicine, Osaka University Graduate School of Medicine, Suita, Japan. 'Division of Nephrology and Hypertension, Yokohama City University Medical Center, Yokohama, Japan. ${ }^{8}$ Department of Medical Science and Cardiorenal Medicine, Yokohama City University Graduate School of Medicine, Yokohama, Japan. ${ }^{9}$ Department of Endocrinology, Metabolism and Nephrology, Keio University School of Medicine, Tokyo, Japan. ${ }^{10}$ Cardiovascular Center, Steel Memorial Yawata Hospital, Fukuoka, Japan. ${ }^{11}$ Health Administration Center, University of the Ryukyus, Okinawa, Japan. ${ }^{12}$ Department of Preventive Medicine and Public Health, Faculty of Medicine, Fukuoka University, Fukuoka, Japan.

${ }^{13}$ Department of Cardiovascular Medicine, Graduate School of Medicine, Nephrology and Neurology, University of the Ryukyus,

Okinawa, Japan. ${ }^{14}$ Tohoku Institute for Management of Blood

Pressre, Sendai, Japan. ${ }^{15}$ Department of Public Health, Shiga University of Medical Science, Otsu, Japan. ${ }^{16}$ Department of Public Health, Okayama University Graduate School of Medicine, Dentistry, and Pharmaceutical Sciences, Okayama, Japan. ${ }^{17}$ Department of Hygiene and Public Health, Teikyo University School of Medicine, Tokyo, Japan. ${ }^{18}$ Tohoku Institute for Management of Blood Pressure, Sendai, Japan. ${ }^{19} \mathrm{KU}$ Leuven Department of Cardiovascular Sciences, University of Leuven, Leuven, Belgium. ${ }^{20}$ Department of Cardiovascular Medicine, Saga University, Saga, Japan. ${ }^{21}$ Division of Cardiovascular Medicine, Department of Medicine, Jichi Medical University School of Medicine, Tochigi, Japan. ${ }^{\bowtie}$ email: yyano@jichi.jp

\section{REFERENCE}

1. Forouzanfar MH, Liu P, Roth GA, Ng M, Biryukov S, Marczak L, et al. Global burden of hypertension and systolic blood pressure of at least 110 to $115 \mathrm{~mm} \mathrm{Hg}$, 1990-2015. JAMA. 2017;317:165-82. 
COMPETING INTERESTS

The authors declare no competing interests.

ADDITIONAL INFORMATION

Correspondence and requests for materials should be addressed to Y.Y.
Reprints and permission information is available at http://www.nature.com/ reprints

Publisher's note Springer Nature remains neutral with regard to jurisdictional claims in published maps and institutional affiliations. 